\title{
Editorial
}

\section{Is aortic valve resistance more clinically meaningful than valve area in aortic stenosis?}

The concept of valve resistance was introduced as a "stenotic index" in the $1950 \mathrm{~s},{ }^{1}$ but it did not reach the worldwide acceptance obtained in the same years by the Gorlin formula to calculate valve area. In more recent years this relatively old concept has been restored as a method for assessing the severity of aortic stenosis. ${ }^{23}$ Aortic valve resistance is simply the pressure gradient/flow rate ratio expressed in units of dyne.s. $\mathrm{cm}^{-5}$. Aortic valve resistance is commonly calculated using cardiac catheterisation with the following equation:

$(1.333 \times \mathrm{P}) \div(\mathrm{CO} / \mathrm{HR} \times \mathrm{SEP})$

where $\mathrm{CO}$ is cardiac output $(\mathrm{ml} / \mathrm{min}), \mathrm{HR}$ is heart rate (beats/min), SEP is systolic ejection period ( $\mathrm{s} / \mathrm{beat}$ ), and 1.333 is the conversion factor from $\mathrm{mm} \mathrm{Hg}$ to dyne.s. $\mathrm{cm}^{-5}$. Aortic valve resistance can also be accurately measured by Doppler echocardiography, using the following formula:

$1.333 \times 4 \mathrm{Vmax}^{2} /$ area LVOT $\times$ velocity LVOT

where $\mathrm{V}$ is the maximum velocity recorded across the aortic valve by continuous wave Doppler, area LVOT is the area of the left ventricular outflow tract obtained from the parasternal long axis view as $3.14 \times$ (diameter/2) assuming a circular shape, and velocity LVOT is the maximum velocity recorded in the left ventricular outflow tract by pulsed wave Doppler.

There are two main reasons for the renewed interest in measuring aortic valve resistance: valve resistance represents a functional index of haemodynamic impairment rather than an anatomic index such as valve area; aortic valve resistance appears to remain more constant as flow varies than calculated aortic valve area-this is particularly relevant in low flow states, in which the valve area calculation may be inaccurate. ${ }^{23}$ Nevertheless, some issues remain:

- is aortic valve resistance really less flow dependent than aortic valve area?

- does aortic valve resistance really provide additional physiopathological and clinical information to valve area and gradient?

- are there reliable cut off values of severity for the routine use of aortic valve resistance in clinical practice?

Flow dependence of aortic valve resistance and aortic valve area

Although transvalvar pressure gradient may be considered the most direct index of aortic stenosis severity, it is well recognised that gradient alone is an inadequate measure of aortic stenosis. ${ }^{23}$ The pressure drop is strictly dependent on the actual flow across the valve; if cardiac output is low, particularly in the presence of left ventricular dysfunction, even a mild gradient may imply severe stenosis. Oh and colleagues $^{4}$ observed that a mean aortic gradient $>50 \mathrm{~mm} \mathrm{Hg}$ was highly specific (94\%) but poorly sensitive $(48 \%)$ for identifying patients with severe aortic stenosis. They even noticed a patient who had severe aortic stenosis (valve area $0.6 \mathrm{~cm}^{2}$ ) and left ventricular ejection fraction $24 \%$ with a measured peak Doppler velocity of only $2.1 \mathrm{~m} / \mathrm{s}$ and a catheterisation derived mean gradient of $8 \mathrm{~mm} \mathrm{Hg}$. On the basis of these observations it has become clear that pressure gradient as a measure of stenosis needs a flow correction. Indeed, both valve area and resistance calculations incorporate cardiac output into the formula. The real problem is which "flow corrected" index could better describe the severity of the aortic stenosis independent of flow conditions?

Several studies have suggested that valvar resistance may be less affected than orifice area by changes in flow, but this is still controversial. Martin et al observed that aortic valve resistance was less influenced by exercise induced increase in cardiac output than Gorlin valve area as measured by cardiac catheterisation. ${ }^{5}$ Burwash et al found a similar flow dependence for aortic valve area and resistance evaluated by Doppler echocardiography during exercise. ${ }^{6}$ Other studies assessed changes in valve area and resistance during dobutamine administration by means of Doppler echocardiography. In these studies valve area was more flow dependent than valve resistance. ${ }^{78}$ Similarly, Lee et al showed that catheterisation derived aortic valve area was more flow dependent than aortic valve resistance in patients with combined aortic and mitral stenosis after percutaneous transvenous balloon dilatation of the mitral valve. ${ }^{9}$ Voelker et al, using a pulsatile aortic flow model and rigid stenotic orifices, demonstrated in vitro that calculated area was less flow dependent than valve resistance. ${ }^{10}$ It is important to consider that in vivo the stenotic aortic valve orifices are not always rigid and so the results of this last study must be considered with caution. Indeed it has recently been observed that the degree of flow dependence of valve area is strictly related to structural characteristics and morphology of the diseased valve. ${ }^{11}$

Most studies indicate a lower flow dependence of aortic valve resistance although this is calculated from the same data as valve area (essentially flow and transvalvar pressure gradient). Actually the main difference between these two indexes is that in calculated area the pressure gradient is proportional to the square of flow and in resistance formula the pressure gradient is proportional to the first power of flow

$\left(\right.$ resistance $=$ gradient $/$ flow; area $=$ flow $/ \mathrm{k} \times$ gradient $t_{1} /$ ) gradient.

Therefore, it is reasonable to assume that flow affects the valve area calculation more than the resistance calculation. Furthermore, it has been demonstrated that the constant employed in the Gorlin formula is not really constant, but varies with blood viscosity, turbulence, gradient, and flow changes. $^{2}{ }^{312}$ Aortic valve resistance calculation does not require any empirical constant: it is simply the gradient divided by flow (the constant 1.333 is just the conversion factor to units of dyne.s. $\mathrm{cm}^{-5}$ ).

\section{Additional physiopathological and clinical} information provided by aortic valve resistance The additional clinical usefulness of valve resistance in the evaluation of the aortic stenosis severity has recently been challenged by Roger et al. ${ }^{13}$ In this study (of 407 patients with aortic stenosis who had Doppler echocardiography before surgery), aortic valve resistance and area were closely related, but valve resistance was not superior to valve area and gradient in describing stenosis severity as assessed by surgical inspection. Furthermore, surgical 


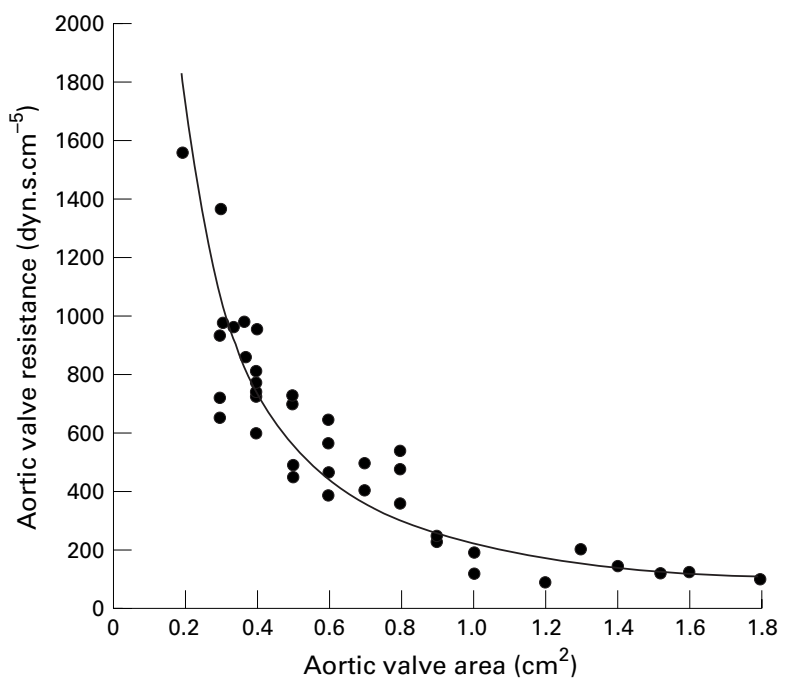

Figure 1 Inverse curvilinear relation between aortic valve area and aortic valve resistance. The slope is steeper with increasing aortic stenosis severity. This could explain why resistance may provide additional clinical information in severe aortic stenosis, when symptoms generally occur. Conversely it could give less information than aortic valve area in mild or moderate aortic stenosis.

mortality was not more related to valve area than aortic valve resistance. This study has two noteworthy limitations: the assessment of stenosis severity by surgical inspection as gold standard is essentially rough; and the number of operative deaths (19) is too small to draw any conclusions.

Other studies indicate some potential clinical usefulness of aortic valve resistance over orifice area and gradient. Cannon et al noticed that aortic valve resistance was more useful than the Gorlin area in separating truly critical valve stenosis from less severe disease in patients with low cardiac output and low gradient. ${ }^{2}$ Isaaz and colleagues ${ }^{14}$ demonstrated that changes in wall stress after valvuloplasty were closely related to changes in valve resistance but not to changes in valve area. In an echocardiographic Doppler study Wang et al observed that aortic valve resistance was related to left ventricular hypertrophy while continuity equation area was not. ${ }^{15}$ Our preliminary data ${ }^{16}$ seem to confirm an independent role for aortic valve resistance in the functional assessment of aortic stenosis severity. In 359 patients with aortic stenosis we observed that catheterisation derived aortic valve resistance correlated better than the Gorlin aortic area with clinical symptoms.

Certainly the hypothesis of an additional clinical role for valve resistance over the valve area needs confirmation, but it is interesting to observe that in the curvilinear relation between these two indexes (fig 1) the slope becomes steeper with increasing severity of aortic stenosis. This implies that in severe aortic stenosis small changes in area may cause large changes in resistance, while in mild and moderate aortic stenosis a little change in resistance may occur despite significant changes in valve area. From a practical point of view this could explain why resistance may provide useful clinical information in patients with severe disease. On the other hand, the measure of aortic valve resistance should be used with caution in the follow up of patients with mild or moderate disease, because in these patients resistance alone might underestimate the progression of stenosis severity over time.
Routine use of aortic valve resistance: a need for reliable cut off values

Despite several encouraging observations on the clinical usefulness of aortic valve resistance, most cardiologists traditionally continue to judge the severity of aortic stenosis on the basis of gradient and calculated valve area. Thus, for these indexes the cut off values of severity are well established and defined for clinical decision making. This is not true for a relatively new index such as aortic valve resistance, which has not been routinely used in clinical practice. In different series a cut off value of 300 dyne.s. $\mathrm{cm}^{-5}$ allowed identification of patients with severe aortic stenosis with good specificity and sensitivity, ${ }^{313}{ }^{16}$ but further clinical studies are needed to validate cut off values of aortic valve resistance with valve area.

As aortic valve resistance can easily be calculated by Doppler echocardiography, ${ }^{7813} 14$ it could be important that cardiologists become familiar with this functional index of haemodynamic impairment and with its units of dyne.s. $\mathrm{cm}^{-5}$.

\section{F ANTONINI-CANTERIN \\ P FAGGIANO}

D ZANUTTINI

Divisione di Cardiologia, ARC,

Azienda Ospedaliera S. Maria degli Angeli, 33170 Pordenone, Italy

F RIBICHINI

Divisione di Cardiologia,

Azienda Ospedaliera S Croce,

Cuneo, Italy

Dr Zanuttini died since this article was submitted for publication.

1 Dow JW, Levine HD, Elkin M, et al. Studies of congenital heart disease, IV: uncomplicated pulmonic stenosis. Circulation 1950;1:267-82.

2 Cannon JD, Zile MR, Crawford FA, et al. Aortic valve resistance as an Cannon JD, Zile MR, Crawford FA, et al. Aortic valve resistance as an
adjunct to the Gorlin formula in assessing the severity of aortic stenosis in adjunct to the Gorlin formula in assessing the severity of a

3 Fymptomatic patients. F Am Coll Cardiol 1992;20:1517-23.

3 For

4 Oh JK, Taliercio CP, Holmes DR, et al. Prediction of the severity of aortic stenosis by Doppler aortic valve area determination: prospective Dopplercatheterization correlation in 100 patients. $\mathcal{F} \mathrm{Am}$ Coll Cardiol 1988;11: 1227-34.

5 Martin TW, Moody JM, Bird JJ, et al. Effects of exercise on indices of valvular aortic stenosis. Cathet Cardiovasc Diagn 1992;25:265-71.

6 Burwash IG, Pearlman A, Kraft CD, et al. Flow dependence of measures of aortic stenosis severity during exercise. 7 Am Coll Cardiol 1994;24:1342-50.

7 deFilippi CR, Willett DL, Brickner ME, et al. Usefulness of dobutamine echocardiography in distinguish severe from nonsevere valvular aortic stenosis in patients with depressed left ventricular function and low transvalvular gradients. Am $\mathcal{F}$ Cardiol 1995;75:191-4.

8 Bermejo J, Garcia-Fernandez A, Torrecilla EG, et al. Effects of dobutamine on Doppler echocardiographic indexes of aortic stenosis. $\mathcal{f} \mathrm{Am}$ Coll Cardiol 1996;28:1206-13.

9 Lee TM, Su SF, Chen MF, et al. Effects of increasing flow rate on aortic stenotic indices: evidence from percutaneous transvenous balloon dilatastenotic indices: evidence from percutaneous transvenous balloon dilata-
tion of the mitral valve in patients with combined aortic and mitral stenotion of the mitral valve in
sis. Heart 1996;76:490-4.

10 Voelker W, Reul H, Nienhaus G, et al. Comparison of valvular resistance, stroke work loss, and Gorlin area for quantification of aortic stenosis. An in vitro study in a pulsatile aortic flow model. Circulation 1995;91:1196-204.

11 Shively BK, Charlton GA, Crawford $\mathrm{MH}$, et al. Flow dependence of valve area in aortic stenosis: relation to valve morphology. $\mathcal{F} \mathrm{Am}$ Coll Cardiol 1998;31:654-60

12 Cannon SR, Richards KL, Crawford M. Hydraulic estimation of stenotic orifice area: a correction of the Gorlin formula. Circulation 1985;71:11708.

13 Roger VL, Seward JB, Bailey KR, et al. Aortic valve resistance in aortic stenosis. Doppler echocardiographic study and surgical correlation. Am Heart f 1997;134:924-9.

14 Isaaz K, Munoz L, Pots T, et al. Demonstration of postvalvuloplasty hemodynamic improvement in aortic stenosis based on Doppler measurement of valvular resistance. $7 \mathrm{Am}$ Coll Cardiol 1991;18:1661-70.

15 Wang Z, Cooke R, Hayes A, et al. Left ventricular mass correlates better with Wang $Z$, Cooke $\mathrm{R}$, Hayes A, et al. Left ventricular mass correlates better with
valve resistance than effective orifice area in aortic stenosis [abstract]. Heart 1996;75(suppl 1):P20.

16 Antonini-Canterin F, Ribichini F, Faggiano P, et al. Comparison between aortic valve resistance and orifice area in assessing aortic stenosis severity
[abstract]. $\mathcal{F}$ Am Coll Cardiol 1998;31(suppl A):324. 